\title{
Ionic liquid simulations: Classical force field fails in structure description of $\left[\mathrm{C}_{4} \mathrm{C}_{1} \mathrm{Im}\right]\left[\mathrm{BF}_{4}\right]$
}

\author{
Kalil Bernardino (PQ) ${ }^{1 *}$ Vitor H. Paschoal $(\mathrm{PG}),{ }^{1}$ Mauro C. C. Ribeiro (PQ) ${ }^{1}$ \\ *kalil.bernardino@gmail.com \\ ${ }^{1}$ Universidade de São Paulo, Instituto de Química \\ Keywords: ionic liquids, force field, molecular dynamics, free energy, parametrization
}

\section{Introduction}

Room temperature ionic liquids presents a set of unique properties interesting for applications as solvents and lubricants, like very high boiling points, great chemical stability and, since most of ionic liquids presents an apolar portion in the cation structure, are able to dissolve both polar and apolar solutes. Computer simulations are useful in order to explain several properties of ionic liquids and to describe its structure and dynamics at a molecular level. One of the most popular force fields employed for ionic liquids is the CL\&P, ${ }^{1}$ which is based in OPLS-AA and shows good agreement for properties like density and expansion coefficient, but overestimate the viscosity. The overestimation of the viscosity is usually solved by the introduction of polarizability or by the used of fractional charges for the ions. However, to the best of our knowledge, other alternatives, like to renounce the combination rules for the Lennard-Jones parameters between different atomic types, where not tried yet. In the present study, a different strategy will be employed for the parametrization of 1-butyl-3-methylimidazolium tetrafluoroborate, $\left[\mathrm{C}_{4} \mathrm{C}_{1} \mathrm{Im}\right]\left[\mathrm{BF}_{4}\right]$, in order to reproduce the structure and the thermodynamics of gas phase dimer, where discrepancies were also found between the force field and $a b$ initio calculations. The refined parameters for the gas phase dimer will then be tested for the properties of the liquid.

\section{Methodology}

Molecular dynamics simulations of the liquid at $T=300 \mathrm{~K}$ and $P=1$ bar were performed using Gromacs 5.1.2 $2^{2}$ suite with the popular CL\&P force field version $2018 / 01 / 05 .{ }^{1}$ The dimer formed by these ions were optimized in vacuum using both the force field parameters in Gromacs and at both MP2/6-31G(p,d), B3LYP/6-31G(p,d) and CAM-B3LYP/Def2-TZVP(-f) theory level using the software Orca 4.0.0.2. ${ }^{3}$ The free energy surface for the translation of the anion close to the cation ring were calculated with both CL\&P force field and at CAM-B3LYP/Def2-TZVP(-f) theory level by means of the numerical calculation of a partition function for the dimer in gas phase.

\section{Results and Discussion}

The free energy surface for the anion over a sphere of radius $3.88 \AA$ around the imidazolium nitrogen N3 is given in Figure $1 \mathbf{a}$, for classic, and $\mathbf{b}$, for quantum descriptions. At each of the 92 points over the translational grid, 25 rigid-body rotations of $\mathrm{BF}_{4}^{-}$were performed and, for each rotation, 5 precessions. Single point calculations were done for each of those structures except the ones with very repulsive contacts using both forcefield parameters and CAM-B3LYP calculations in order to determine the interaction energy for each structure. Canonical partition functions where produced with those energies in each translational point and thermodynamic properties were derived from those. The red points in the Figure $1 \mathbf{a}$ and $\mathbf{b}$ corresponds the the lowest free energy values found and a significant difference arises: While the quantum calculation shows the most favorable position for the $\left[\mathrm{BF}_{4}\right]^{-}$as close to the hydrogen 2 of the cation ring, with one of the fluorine atoms forming a hydrogen bond with the 
cation, the CL\&P force field predicts lower free energies for the anion above and below the ring.

As expected by the free energy surfaces constructed with rigid rotations, the force field also fails in the fully optimization of the dimer, as showed by the structures in Figure $1 \mathrm{c}$, where the red was obtained with CL\&P parameters and the black one from the MP2 calculation starting with the final structure from the classical optimization. In the MP2 the anion moves toward the hydrogen attached to carbon C2, with the distance between this hydrogen and the closest fluorine atom of $2.0 \AA$, which is in the upper limit of which is considered as a hydrogen bond. This tendency observed for the gas phase dimer may affect the simulations of the liquid with the force field, where the first peak of radial distribution function between these atoms occur only at $2.5 \AA$, out of the range of usual hydrogen bonds. B3LYP and CAM-B3LYP optimizations result in structures very similar to the one showed here for MP2.
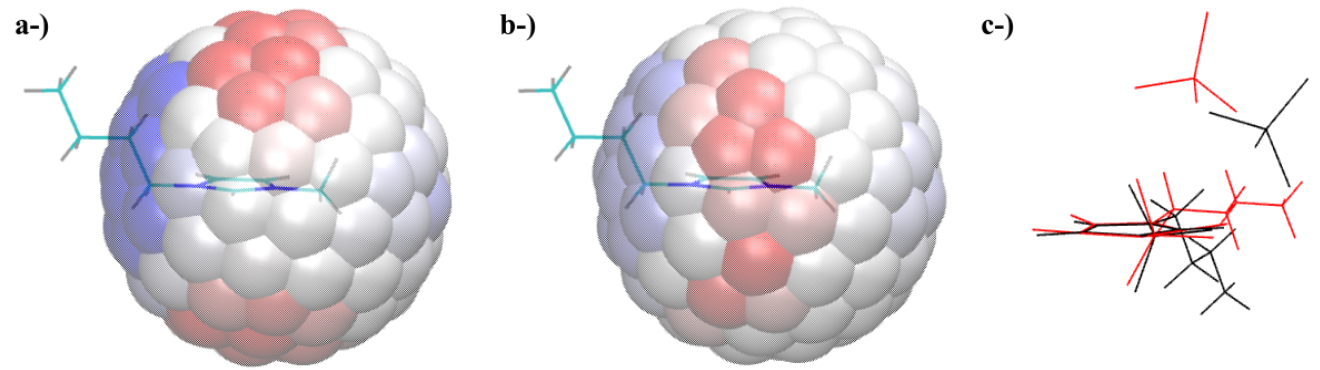

Figure 1 - a-) Free energy surface for the $\left[\mathrm{BF}_{4}\right]^{-}$around the imidazolium N3 described by CL\&P force field. b-) Free energy surface for the anion around N3 described by CAM-B3LYP calculation. c-) Fully optimized structure of the dimer in the quantum (black) and force field (red) calculations. In $\mathbf{a}$ and $\mathbf{b}$, the blue color correspond to the grid points with highest free energy values while the red to the lowest ones.

\section{Conclusions}

Even with a good description of macroscopic properties, the microscopic structure predicted by a force field may be wrong. Comparison with quantum calculations and, if available, diffraction data of the liquid phase, must be done in order to validate a force field. The non-bonded parameters of the force field will be recalculated in order to adjust the free energy surface of the dimer obtained in the with the CAM-B3LYP functional. Introduction of polarizability and fractional total charge for the ions will also be tested and static and dynamic properties of the liquid will be evaluated for each improved set of parameters obtained.

\section{Acknowledgments}

We thank FAPESP for financial support and scholarships (grant numbers 2017/12063-8, 2016/21070-5 and 2015/07516-8).

\section{References}

1- Shimizu, K.; Almantariotis, D.; Gomes, M. F. C.; Pádua, A. A. H.; Lopes, J. N. C.. J. Phys. Chem. B, 2010, 114 (10), pp 3592-3600.

2- Berendsen, H. J. C.; van der Spoel, D.; van Drunen, R. Comp. Phys. Comm., 1995, $91:$ 43-56.

3- Neese, F.; Wiley Interdisciplinary Reviews: Computational Molecular Science., 2012, 2 (1): 73-78. 